\title{
High Spatial Resolution Observations of the 1998 and 1999 Leonid Meteors
}

\author{
X.J. Jiang ${ }^{1,2}$ and J.Y. $\mathrm{Hu}^{1,2}$ \\ 1. Beijing Astronomical Observatory, Chinese Academy of Sciences, \\ Beijing, 100012, P.R. China \\ 2. National Astronomical Observatories, Chinese Academy of Sciences, \\ Beijing 100012, China
}

Abstract. We observed the Leonid meteor storm in 1998 and 1999. The light curves extracted from the 2-dimensional images of the bright Leonid meteors clearly show the sub-structures, which are consistent with the generally accepted dustball meteor model (Hawkes \& Jones, 1975).

\section{Introduction}

During November 17-19 of each year, the Earth passes through the debris cloud from comet Temple-Tuttle. This event generally creates a meteor shower as the particles enter the Earth's atmosphere and burn up. Since the debris appears to be coming from the direction of the constellation Leo, it is called the "Leonid shower".

Every 33 years, the Earth passes very close to Comet Temple-Tuttle's orbital path not long from when the comet has recently passed by. As a result, the Earth is likely to experience a meteoroid "storm" as it passes through the cloud of debris following closely behind the comet. The most recent Leonid meteor storm arises between 1998-2001, which provides a good opportunity to study the cometary originated meteoroids.

\section{Observations and Discussions}

Figure 1 shows the meteor light curves. Panels a,b,and $c$ were taken on November 18, 1998 at XingLong Station, Beijing Astronomical Observatory with a 16-bit TEK $1024 \mathrm{CCD}$ and a Carl Zeiss Jena Schmidt-Cassegrain camera (f.l. $150 \mathrm{~mm}$, $\mathrm{f} / 1.5$ ). The center of each field is about $10^{\circ}$ away from the Leonid radiant, the field of view of the raw CCD image is $9.4^{\circ} \times 9.4^{\circ}$, and the resolution is $33^{\prime \prime} / \mathrm{pixel}$. The exposure time was fixed to 300 seconds. Panel $\mathrm{c}$ was taken on November 18,1999 with SBIG ST-8 CCD and a $50 \mathrm{~mm} \mathrm{f} / 1.8$ lens, The field of view of the raw image is $15.8^{\circ} \times 10.6^{\circ}$, and the resolution is $37^{\prime \prime} / \mathrm{pixel}$.

The raw data were reduced by using the IRAF program package. The CCD reductions included bias subtraction and flat field correction, then the light curves were extracted from the two-dimensional images. 

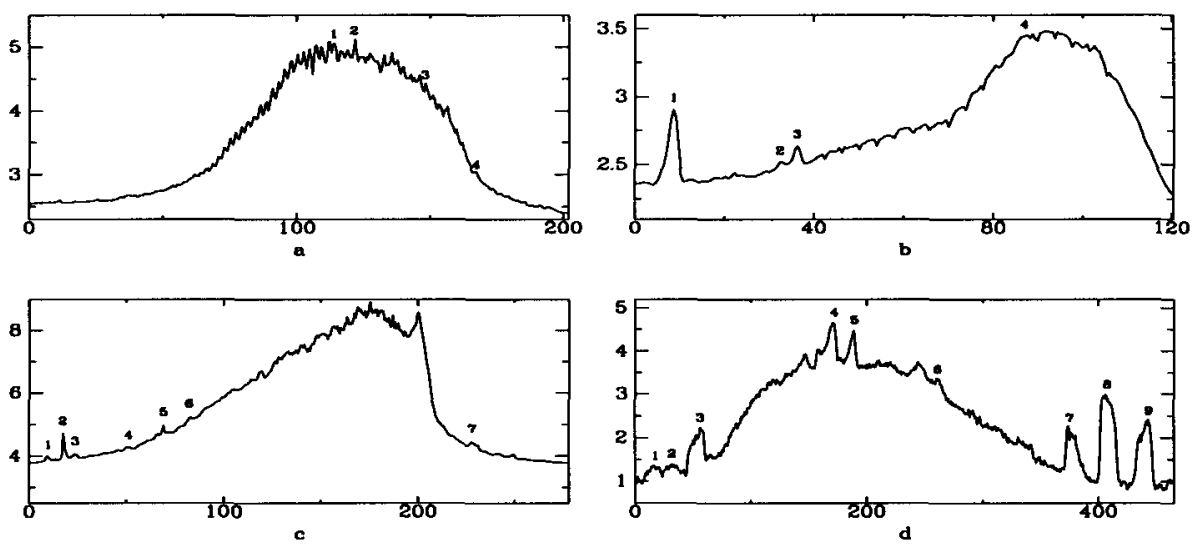

Figure 1. Light curves of the four bright Leonid meteors. The relative intensity is plotted against the trajectory in arc minutes. The numbered peaks were caused by the background stars

Since all the images were taken by fixing the CCD camera on a camera tripod, some background star trails overlaid on the meteor's light curves. We found that it is very difficult to remove the background stars from the light curves without leaving artificial marks, so we decided to leave all the star trails and mark them on the light curves.

Besides the features caused by the background stars, there are still many sub-structures on the light curves. We consider that the rises and falls are due to the intrinsic properties of the meteoroids, i.e., the silicate and metallic grains described in the dustball model.

To confirm the idea, we first measured the intensities of these features, finding that they are much higher than the noise level of the light curves. Then we extracted random sky backgrounds from the original images, finding that the rises and falls in the sky backgrounds are much lower in intensity and less in density compared with the features that appeared on the meteor's light curves, which means these features are not from the background sky.

The typical flux of the split particles is about $0.1 \%$ of the total flux of the host meteoroid, implying that the mass of these particles is typically $0.1 \%$ of the whole meteoroid. We also inspected all the four images. No evidence of transverse spread or jet-like features as observed by LeBlanc et al (2000) was found, though our spatial resolution and detector's dynamic range are higher.

\section{References}

Hawkes, R.L., Jones, J. 1975, MNRAS, 173, 339

LeBlanc, A.G., Murray, I.S., Hawkes, R.L., Worden, P., Campbell, M.D., et al. 2000, MNRAS, 313, L9

Monet, D. et al. 1999, USNO-A2.0 (Washington: U.S. Naval Observatory) 